\title{
Stabilization of flavylium dye by incorporation into Fe-containing mesoporous silicate
}

\author{
Yoshiumi Kohno ${ }^{a,}$, Masanori Senga ${ }^{a}$, Masashi Shibata ${ }^{b}$, Keiko Yoda ${ }^{c}$, Ryoka \\ Matsushima $^{a}$, Yasumasa Tomita ${ }^{a}$, Yasuhisa Maeda ${ }^{a}$, Kenkichiro Kobayashi ${ }^{a}$ \\ ${ }^{a}$ Department of Materials Science and Engineering, Faculty of Engineering, Shizuoka University, 3-5-1 \\ Johoku, Naka-ku, Hamamatsu, Shizuoka, 432-8561, Japan \\ ${ }^{b}$ School of Bioscience and Biotechnology, Tokyo University of Technology, 1404 Katakura-machi, \\ Hachioji, Tokyo 192-0982, Japan \\ ${ }^{c}$ Beauty Research Center, Kao Corp., 2-1-3 Bunka, Sumida-ku, Tokyo 131-8501, Japan \\ *corresponding author e-mail: tykouno@ipc.shizuoka.ac.jp, phone:+81-53-478-1623
}

\begin{abstract}
Flavylium dye, an analogue of natural anthocyanin, is an expectedly safe coloring material. However, their color instability limits its practical use. We have prepared Fe-containing HMS type mesoporous silicate, and have made a composite of the FeHMS and the flavylium dye. The color stability of the flavylium under visible light irradiation has been enhanced by making the composite with the FeHMS. During $8 \mathrm{~h}$ irradiation, $45 \%$ of the absorption of the flavylium adsorbed on HMS has been lost, while only $5 \%$ of the absorption has been lost on the FeHMS with 2 mol \% Fe. From the UV-vis spectra, FeHMS has been confirmed to contain a $\mathrm{Fe}^{3+}$ species highly dispersed in the silica framework. Since the $\mathrm{Fe}^{3+}$ species works as an acid site in the mesopore of HMS, the enhancement of the stability has partially been attributed to the solid acidity of the FeHMS. Under strong light irradiation (> $800 \mathrm{klx}$ ), the degradation rate of the flavylium has been independent of the light irradiance, because the diffusion rate of the oxygen into the mesopore cannot satisfy the demand to oxidize the flavylium dye under irradiation. This means that the inhibition of the contact with the external oxygen also contributes to the stabilization of the flavylium dye incorporated in the mesopore.
\end{abstract}

keywords: Flavylium, Anthocyanin, Stabilization, Light fastness, Mesoporous silica 


\section{Introduction}

Flavylium dye is a derivatives of 2-phenylbenzopylilium, a chromophore common to a naturally occurring anthocyanin. Therefore, the flavylium is considered as a synthetic model compound of the anthocyanin [1-3]. The anthocyanin is known to be a non-toxic material and widely used as a food colorant [4]. The flavylium is also expected to be less toxic because it has the same skeleton as the anthocyanin [5-7]. The less-toxicity of the flavylium will urge the wide usage as a safe colorant. However, the flavylium has fatal disadvantage that the flavylium is quite unstable unless kept in acidic media. Under neutral to slightly basic condition, its color quickly fades away [8]. Such a feature of instability is also common in the naturally occurring anthocyanin [9-11].

There have been some reports trying to stabilize natural dyes using inorganic host materials [12-16]. The stability of the flavylium can be enhanced by making a composite with several inorganic hosts such as clays [17], zeolites [18] or mesoporous silicas [19]. All these materials have so-called nano-space because they have layered or porous structure. In agreement with other studies [12, 20-24], the dye molecules are stabilized by incorporation into the nano-space. The causes of the stabilization are both the solid acidity of the inorganic hosts and the shielding effect from the external circumstances.

In this study, we report the stabilization of the flavylium dye by complexation with Fe-containing mesoporous silicate. Previous study revealed that the introduction of $\mathrm{Al}^{3+}$ to mesoporous silicas forms acid sites, resulting in the sufficient stabilization of the flavylium [19]. Since the introduction of $\mathrm{Fe}^{3+}$ into tectosilicate is also reported to lead the appearance of acid site [25-30], the Fe-containing mesoporous silica is expected to exhibit the effect of the stabilization on the incorporated flavylium. We adopt HMS type mesoporous silicas with randomly ordered mesopores instead of the ones with highly ordered hexagonal pores such as MCM-41 or FSM-16 in this study, because a long-range regularity of the mesopore is not required for the stabilization of the molecules [19]. This study is focused on the detailed clarification of the relationship between the stabilization effect of the flavylium and the state or the amount of the Fe species in the mesoporous silica. In addition, it is confirmed that the incorporation of the flavylium into the mesopore is essential for the stabilization. 


\section{Experimental}

\subsection{Sample preparation}

HMS was prepared based on the literature [31]. Dodecylamine weighed $0.91 \mathrm{~g}$ (Wako Chem.) was dissolved in $5 \mathrm{~cm}^{3}$ of ethanol, mixed with $45 \mathrm{~cm}^{3}$ of water, and heated up to $333 \mathrm{~K}$. Tetraethoxysilane (Wako Chem., denoted as TEOS) weighed $4.6 \mathrm{~g}$ was dropped to the solution, and the mixture was kept at $333 \mathrm{~K}$ for $22 \mathrm{~h}$. The sample was filtered, washed with water, dried in an oven and calcined at $903 \mathrm{~K}$ for $6 \mathrm{~h}$ under dry air stream. The molar composition was 1.0 dodecylamine : 4.5 TEOS : $511 \mathrm{H}_{2} \mathrm{O}: 17$ ethanol.

To prepare the Fe-containing HMS, either $\mathrm{Fe}\left(\mathrm{NO}_{3}\right)_{3} \cdot 9 \mathrm{H}_{2} \mathrm{O}$ (Wako Chem.) or tris(2,4-pentanedionato)iron(III), (Dojindo Chem., denoted as Fe(acac) 3 ), was used as a source compound of $\mathrm{Fe}^{3+}$. On using $\mathrm{Fe}\left(\mathrm{NO}_{3}\right)_{3}$ as an iron source, given amount of the $\mathrm{Fe}\left(\mathrm{NO}_{3}\right)_{3}$ was dissolved in $5 \mathrm{~cm}^{3}$ of water, and the solution was added drop by drop to the dodecylamine solution with TEOS by turns. On the other hand, Fe(acac) $)_{3}$ was dissolved in $3 \mathrm{~cm}^{3}$ of ethanol, mixed with TEOS, and then poured onto the dodecylamine solution. After mixing the source compounds, the preparation procedure was the same as that of pure HMS described above. In the following, the Fe-containing HMS is referred to as $\mathrm{Fe}(\mathrm{N} x) \mathrm{HMS}$ or $\mathrm{Fe}(\mathrm{A} x) \mathrm{HMS}$, where $\mathrm{N}$ and A denotes the $\mathrm{Fe}$ sources $\left(\mathrm{N}: \mathrm{Fe}\left(\mathrm{NO}_{3}\right)_{3}, \mathrm{~A}\right.$ : $\left.\mathrm{Fe}(\mathrm{acac})_{3}\right)$, and $x$ represents the $\mathrm{Fe} / \mathrm{Si}$ molar ratio of the starting mixture expressed in percentage.

The prepared HMS and FeHMS samples were characterized by XRD and nitrogen adsorption. In the XRD pattern, all the samples showed a single reflection peak at around $2 \theta=2^{\circ}$. The BET specific surface areas of typical samples were greater than $800 \mathrm{~m}^{2} / \mathrm{g}$. Those samples exhibited a narrow pore size distribution with the mean pore diameters in the range between 2.8 and $3.0 \mathrm{~nm}$. From these, the samples had a mesoporous structure.

The structure of the flavylium dye (FV) used in this study is shown in Scheme 1. The FV has a methoxy group at 4'-position and a hydroxyl group at 7-position. The FV dye exhibits yellow color in an acidic solution, while it shows red shift of the absorption band in a weakly basic solution. The red shift is due to the dissociation of a proton from the hydroxyl group [19, 32]. 
The complexation of FV with FeHMS was carried out as follows. The FV dye (2 $\mathrm{mg}$ ) was dissolved in a $50 \mathrm{~cm}^{3}$ of aqueous ethanol solution (water : ethanol $=7: 3$ ), and $200 \mathrm{mg}$ of the FeHMS was mixed with the solution. The mixture was kept in the dark for 3 days to reach the adsorption equilibrium, followed by filtration to separate the composite sample. The separated powder composite was dried at $353 \mathrm{~K}$ in the air. Hereafter, the composite samples are referred to as FV/FeHMS. As a reference sample for comparison of the light fastness, the composites of FV with HMS and silica were prepared in the same manner as FV/FeHMS. The silica (denoted as SIO) was supplied from Japan Catalyst Society (JRC-SIO-9A). In the following, these composites are referred to as FV/HMS and FV/SIO, respectively.

\subsection{Apparatus and procedures}

Diffuse reflectance UV-Vis spectra were collected with JASCO V-550 spectrophotometer equipped with ISV-469 integrating sphere. On measuring spectra, the powder samples were loaded in a plastic cell covered with a thin glass.

To investigate the light endurance of each sample, visible light was irradiated to the samples under ambient temperature and pressure. A $100 \mathrm{~W}$ halogen lamp (Schott Megalight 100) equipped with a $30 \mathrm{~cm}$ length flexible light guide was used as a visible light source. The powder samples were held in the same cell as that used for the UV-vis measurement. Unless otherwise noted, the samples were placed at the position of $1.1 \mathrm{~cm}$ from the aperture of the light guide, and the light intensity was adjusted to $E=190 \mathrm{klx}$. To investigate the effect of the light intensity, the samples were placed at the position of $0.3 \mathrm{~cm}$ from the aperture, and the light intensity was changed from $200 \mathrm{klx}$ to $1500 \mathrm{klx}$ at the sample position. The degree of the color fading was determined by the decrease in the absorption at $\lambda_{\max }$ in the diffuse reflectance spectra.

\section{Results and discussion}

\subsection{UV-vis spectra of FeHMS}

Figure 1 illustrates the diffuse-reflectance UV-vis spectra of $\mathrm{Fe}(\mathrm{N}) \mathrm{HMS}$ and Fe(A)HMS samples with different amount of Fe loading. Both series of the samples 
showed an absorption peak at around $250 \mathrm{~nm}$, which was assigned to highly dispersed $\mathrm{Fe}^{3+}$ species [25, 29, 33-38]. Fe(N)HMS showed a single peak at $245 \mathrm{~nm}$, while Fe(A)HMS exhibited a couple of peaks at $220 \mathrm{~nm}$ and $255 \mathrm{~nm}$. Highly dispersed tetrahedral (4-coordinated) $\mathrm{Fe}^{3+}$ species shows absorption at around the wavelength region [25, 35-38]. Liu et al. have reported that Fe-containing HMS exhibits a single peak at $260 \mathrm{~nm}$, and they have assigned the absorption at $260 \mathrm{~nm}$ to the highly dispersed tetrahedral $\mathrm{Fe}^{3+}$ species incorporated in the silica framework [35]. On the other hand, Tuel et al. have observed a pair of peaks at around $220 \mathrm{~nm}$ and $250 \mathrm{~nm}$ in their FeHMS, and have assigned them to two transitions involving $\mathrm{Fe}^{3+}$ in $\mathrm{FeO}_{4}^{-}$ tetrahedra [36]. Therefore, the Fe species included in our Fe(N)HMS and Fe(A)HMS were both the tetrahedral $\mathrm{Fe}^{3+}$. However, at this stage we could not entirely exclude the possibility that the species was octahedrally coordinated one [33].

In both $\mathrm{Fe}(\mathrm{N}) \mathrm{HMS}$ and $\mathrm{Fe}(\mathrm{A}) \mathrm{HMS}$, the absorption increased along with the increase in the amount of Fe loading. However, the behavior of the spectra on the increase in the Fe amount was different from each other. As shown in Figure 1[A], the peak at $245 \mathrm{~nm}$ reached its maximum absorption in Fe(N0.25)HMS. In Fe(N0.50)HMS, the absorption was almost identical to that in Fe(A0.25)HMS, but the longer wavelength region at around $400 \mathrm{~nm}$ appeared. The growth of the absorption at around $400 \mathrm{~nm}$ indicates aggregation of the Fe species, resulting in the formation of extra framework iron oxide particles [38]. On the other hand, as shown in Figure 1[B], even in Fe(A2.0)HMS the growth of the absorption at $400 \mathrm{~nm}$ was not observed, suggesting that the sample consisted of the highly dispersed Fe species. The absorption of Fe(A0.50)HMS at around $250 \mathrm{~nm}$ reaches about 2, which is quite larger than that of Fe(N0.25)HMS. Therefore, Fe(A)HMS had more dispersive Fe species than Fe(N)HMS. Indeed, the spectra of Fe(A4.0)HMS showed the formation of the aggregate, too.

\subsection{Spectral changes of FV composites under irradiation}

Next, the durability of the FV composites on visible light irradiation was compared. Figure 2 represents the spectral changes during $8 \mathrm{~h}$ irradiation of FV/HMS and FV/Fe(A2.0)HMS. The absorption peak of FV/HMS obviously decreased along with irradiation. During the decrease in the absorption under irradiation, the spectral shape was almost unchanged, indicating that the fading was due not to the photochemical 
conversion such as isomerization, but to a simple degradation.

On the contrary, the absorption of FV/Fe(A2.0)HMS did not show apparent decrease. In other words, HMS did not show the effect to enhance the stability of FV by making complex, while Fe(A2.0)HMS exhibited the effect on the stabilization of FV. The enhancement of FV stability was also observed to some degree in all FV/FeHMS samples, as well as in FV/Fe(A2.0)HMS. The degree of the stabilization was dependent on the amount of Fe, as discussed later in section 3.3.

The half-bandwidth of FV/Fe(A2.0)HMS was narrower than FV/HMS. This narrowing was mainly due to the disappearance of the shoulder peak observed in FV/HMS at $515 \mathrm{~nm}$. Thus, FV/Fe(A2.0)HMS showed only a single sharp peak at 470 $\mathrm{nm}$. The absorption of FV at $515 \mathrm{~nm}$ can be assigned to the form where the phenolic hydroxyl group of FV is dissociated. In an acidic media, the hydroxyl group is protonated, resulting in the blue shift of the predominant peak [19]. The peak at around $350 \mathrm{~nm}$ was assigned to the corresponding 2-hydroxychalcone form [1, 19].

The FeHMS samples showed a peak at around $250 \mathrm{~nm}$, indicating that FeHMS possessed highly dispersed $\mathrm{Fe}^{3+}$ species. Highly dispersed tetrahedral $\mathrm{Fe}^{3+}$ species exhibits solid acidity in the tectosilicate framework [25-30]. The acid site of the FeHMS caused the blue shift of the absorption peak of FV in FV/Fe(A2.0)HMS. Similar blue shift has also been observed in FV/AlHMS, where the peak shifted to $450 \mathrm{~nm}$ by the acid site formed by the $\mathrm{Al}^{3+}$ [19]. In the FV/FeHMS, the peak was observed at $470 \mathrm{~nm}$. The blue shift observed in FV/FeHMS was rather small compared to FV/AlHMS, probably because the acidity of $\mathrm{Fe}^{3+}$ incorporated in the mesoporous silicate was not as strong as $\mathrm{Al}^{3+}$ [26].

\subsection{Dependency on the amount of Fe species}

Figure 3 depicts the relationship between the amount of $\mathrm{Fe}$ species and the stabilization effect on FV. The ordinate was the ratio of the absorption of FV/FeHMS before and after visible light irradiation for $8 \mathrm{~h}$, and the abscissa was the value of the absorption of various FeHMS samples at around $250 \mathrm{~nm}$, reflecting the amount of highly dispersed $\mathrm{Fe}^{3+}$ species. The data reproducibility was confirmed to be within few percent. A linear relationship was found between the durability of FV and the absorption at around $250 \mathrm{~nm}$. This result strongly suggests that the highly dispersed $\mathrm{Fe}^{3+}$ species 
contributed to the stabilization of FV. As mentioned above, tetrahedral $\mathrm{Fe}^{3+}$ works as an acid site in HMS. Therefore, FeHMS included the tetrahedral $\mathrm{Fe}^{3+}$, and that the resulting acid site adsorbed FV molecules to stabilize them.

On the other hand, the stabilization effect was independent of the source compound of the $\mathrm{Fe}^{3+}$ species. Therefore, both $\mathrm{Fe}\left(\mathrm{NO}_{3}\right)_{3}$ and $\mathrm{Fe}(\mathrm{acac})_{3}$ can be used as a precursor of the stabilizer. However, since the Fe species in Fe(A)HMS was more dispersive than in $\mathrm{Fe}(\mathrm{N}) \mathrm{HMS}$, the maximum amount of the tetrahedral $\mathrm{Fe}^{3+}$ species (i.e., the maximum stabilization effect) was higher in $\mathrm{Fe}(\mathrm{A}) \mathrm{HMS}$ than in $\mathrm{Fe}(\mathrm{N}) \mathrm{HMS}$. Therefore, $\mathrm{Fe}(\mathrm{acac})_{3}$ is a favorable compound to $\mathrm{Fe}\left(\mathrm{NO}_{3}\right)_{3}$ as an iron source of FeHMS.

\subsection{Dependency on the light Intensity}

Figure 4 exhibits the relationship between the light irradiance and the durability of FV in several FV composite samples. In FV/SIO, the decomposition of FV gradually became severe as the light intensity increased. This result suggests that FV molecules were adsorbed on the surface of SIO, and that photoexcited FV was decomposed by oxidation proportional to the increase in the light intensity. On the other hand, in FV/Fe(A0.083)HMS no more enhancement of the FV degradation was observed under irradiation stronger than $800 \mathrm{klx}$. These results can be explained by the idea that the FV molecules were incorporated in the mesopore of FeHMS. Concretely speaking, under strong light irradiation the diffusion rate of $\mathrm{O}_{2}$ molecule could not satisfy the consumption of $\mathrm{O}_{2}$ for the oxidation of FV in the mesopore. This is in agreement with our previous conclusion that the inhibition of the $\mathrm{O}_{2}$ diffusion contributes to the stabilization of FV incorporated in the mesopore [19].

In FV/HMS, the degradation behavior of FV was similar to that in FV/SIO. Namely, the degree of the FV degradation increased monotonously along with the light intensity. This means that the state of the adsorbed FV resembled each other on HMS and on SIO. The FV molecules were not incorporated in the mesopore of HMS but adsorbed simply onto the outer surface. In other words, the $\mathrm{Fe}^{3+}$ species prompted the FV molecules to be incorporated into the mesopore of FeHMS, probably by an electrostatic interaction, because FV was a cationic molecule. Thus, the acid site formed by the $\mathrm{Fe}^{3+}$ species attracted the cationic FV molecules and incorporated them into the mesopore. 
For summary, the $\mathrm{Fe}^{3+}$ site in the HMS framework drove the incorporation of FV molecules into the mesopore. The acidity caused by the incorporated $\mathrm{Fe}^{3+}$ stabilized the FV molecules. At the same time, the inhibition of the diffusion of $\mathrm{O}_{2}$ molecule from the external circumstance was also confirmed to contribute to the stabilization effect. For example, during $8 \mathrm{~h}$ irradiation, the loss of the absorption of FV was 9 times smaller in $\mathrm{FV} / \mathrm{Fe}(\mathrm{A} 2.0) \mathrm{HMS}$ than in FV/HMS.

\section{Acknowledgment}

A part of this work was financially supported by the Cosmetology Research Foundation. 


\section{Figure Captions}

Figure 1 Diffuse-reflectance UV-Vis spectra of [A] Fe(N)HMS and [B] Fe(A)HMS. The Fe/Si ratio was (a) 0.025, (b) 0.083, (c) 0.125, (d) 0.25 and (e) 0.50 in the part [A], and (a) 0.083, (b) 0.50, (c) 1.0, (d) 2.0 and (e) 4.0 in the part [B].

Figure 2 Changes in the diffuse-reflectance UV-Vis spectra of [A] FV/HMS and [B] FV/Fe(A2.0)HMS during visible light irradiation for $8 \mathrm{~h}$. The spectra were measured at the intervals of $2 \mathrm{~h}$.

Figure 3 Relationship between the absorption at $250 \mathrm{~nm}$ in FeHMS and the retention ratio in the absorption of FV in FV/FeHMS before and after visible light irradiation for $8 \mathrm{~h}$. The shape of symbols indicates the difference in the Fe source of FeHMS. Circle: FV/Fe(N)HMS. Square: FV/Fe(A)HMS.

Figure 4 Dependencies of the retention ratio of FV absorption in various composite samples on the intensity of irradiated light. The samples were (a) FV/Fe(A0.083)HMS, (b) FV/SIO and (c) FV/HMS. Irradiation period was $2 \mathrm{~h}$ for FV/Fe(A0.083)HMS, and $0.5 \mathrm{~h}$ for FV/SIO and FV/HMS. 


\section{References}

[1] R.A. McClelland, S. Gedge, J. Am. Chem. Soc. 102 (1980) 5838.

[2] M.C. Moncada, S. Moura, M.J. Melo, A. Roque, C. Lodeiro, F. Pina, Inorg. Chim. Acta. 356 (2003) 51.

[3] M.C. Moncada, M.F. de Mesquita, M.M.C. dos Santos, J. Electroanal. Chem. 636 (2009) 60.

[4] G. Mazza, R. Brouillard, Food Chem. 25 (1987) 207.

[5] G.A. Iacobucci, J.G. Sweeny, Tetrahedron. 39 (1983) 3005.

[6] R. Matsushima, H. Mizuno, H. Itoh, J. Photochem. Photobiol. A. 89 (1995)

251.

[7] F. Ito, N. Tanaka, A. Katsuki, T. Fujii, J. Photochem. Photobiol. A. 150 (2002)

153.

[8] ～L. Jurd, J. Org. Chem. 28 (1963) 987.

[9] C.F. Timberlake, P. Bridle, Nature. 212 (1966) 158.

[10] ～R. Brouillard, J.-E. Dubois, J. Am. Chem. Soc. 99 (1977) 1359.

[11] K. Torskangerpoll, O.M. Andersen, Food Chem. 89 (2004) 427.

[12] T. Itoh, K. Yano, Y. Fukushima, J. Am. Chem. Soc. 124 (2002) 13437.

[13] E. Lima, M.J. Martinez-Ortiz, E. Fregoso, J. Mendez-Vivar, Z.G.J.C. Ruren

Xu, Y. Wenfu, Stud. Surf. Sci. Catal., Elsevier, 2007, pp. 2110.

[14] E. Lima, P. Bosch, S. Loera, I.A. Ibarra, H. Laguna, V. Lara, Appl. Clay Sci. 42 (2009) 478.

[15] Y. Kohno, R. Kinoshita, S. Ikoma, K. Yoda, M. Shibata, R. Matsushima, Y. Tomita, Y. Maeda, K. Kobayashi, Appl. Clay Sci. 42 (2009) 519.

[16] Y. Kohno, K. Totsuka, S. Ikoma, K. Yoda, M. Shibata, R. Matsushima, Y. Tomita, Y. Maeda, K. Kobayashi, J. Colloid Interface Sci. 337 (2009) 117.

[17] Y. Kohno, R. Hoshino, R. Matsushima, Y. Tomita, K. Kobayashi, J. Jpn. Soc. Colour Mater. 80 (2007) 6.

[18] Y. Kohno, Y. Shibata, N. Oyaizu, K. Yoda, M. Shibata, R. Matsushima, Microporous Mesoporous Mater. 114 (2008) 373.

[19] Y. Kohno, S. Tsubota, Y. Shibata, K. Nozawa, K. Yoda, M. Shibata, R. Matsushima, Microporous Mesoporous Mater. 116 (2008) 70. 
[20] Â.A. Teixeira-Neto, A.L. Shiguihara, C.M.S. Izumi, M.A. Bizeto, F. Leroux, M.L.A. Temperini, V.R.L. Constantino, Dalton Trans. (2009) 4136.

[21] T. Saito, K. Fukui, Y. Kodera, A. Matsuyama, H. Nishimura, Y. Inada, Dyes Pigm. 65 (2005) 21.

[22] Z. Gabelica, S. Valange, M. Shibata, H. Hotta, T. Suzuki, Microporous Mesoporous Mater. 44-45 (2001) 645.

[23] M. Shibata, H. Hotta, T. Suzuki, S. Valange, Z. Gabelica, Chem. Lett. (1999) 1291.

[24] H. Laguna, S. Loera, I.A. Ibarra, E. Lima, M.A. Vera, V. Lara, Microporous Mesoporous Mater. 98 (2007) 234.

[25] Y. Wang, Q. Zhang, T. Shishido, K. Takehira, J. Catal. 209 (2002) 186.

[26] C.T.W. Chu, C.D. Chang, J. Phys. Chem. 89 (1985) 1569.

[27] J. Datka, T. Abramowicz, J. Chem. Soc., Faraday Trans. 90 (1994) 2417.

[28] D.J. Parrillo, C. Lee, R.J. Gorte, D. White, W.E. Farneth, J. Phys. Chem. 99 (1995) 8745.

[29] K. Bachari, J.M.M. Millet, B. Benaïchouba, O. Cherifi, F. Figueras, J. Catal. $221(2004) 55$.

[30] C. Anand, P. Srinivasu, S. Alam, V.V. Balasubramanian, D.P. Sawant, M.

Palanichamy, V. Murugesan, A. Vinu, Microporous Mesoporous Mater. 111 (2008) 72.

[31] N. Marín-Astorga, G. Pecchi, T.J. Pinnavaia, G. Alvez-Manoli, P. Reyes, J. Mol. Catal. A: Chem. 247 (2006) 145.

[32] J.C. Lima, I. Abreu, R. Brouillard, A.L. Maçanita, Chem. Phys. Lett. 298 (1998) 189.

[33] S. Bordiga, R. Buzzoni, F. Geobaldo, C. Lamberti, E. Giamello, A. Zecchina, G. Leofanti, G. Petrini, G. Tozzola, G. Vlaic, J. Catal. 158 (1996) 486.

[34] M. Iwamoto, T. Abe, Y. Tachibana, J. Mol. Catal. A: Chem. 155 (2000) 143.

[35] H. Liu, G. Lu, Y. Guo, Y. Guo, J. Wang, Microporous Mesoporous Mater. 108 (2008) 56.

[36] A. Tuel, I. Arcon, J.M.M. Millet, J. Chem. Soc., Faraday Trans. 94 (1998) 3501.

[37] C. Nozaki, C.G. Lugmair, A.T. Bell, T.D. Tilley, J. Am. Chem. Soc. 124 (2002) 13194. 
[38] V. Umamaheswari, W. Böhlmann, A. Pöppl, A. Vinu, M. Hartmann, Microporous Mesoporous Mater. 89 (2006) 47. 

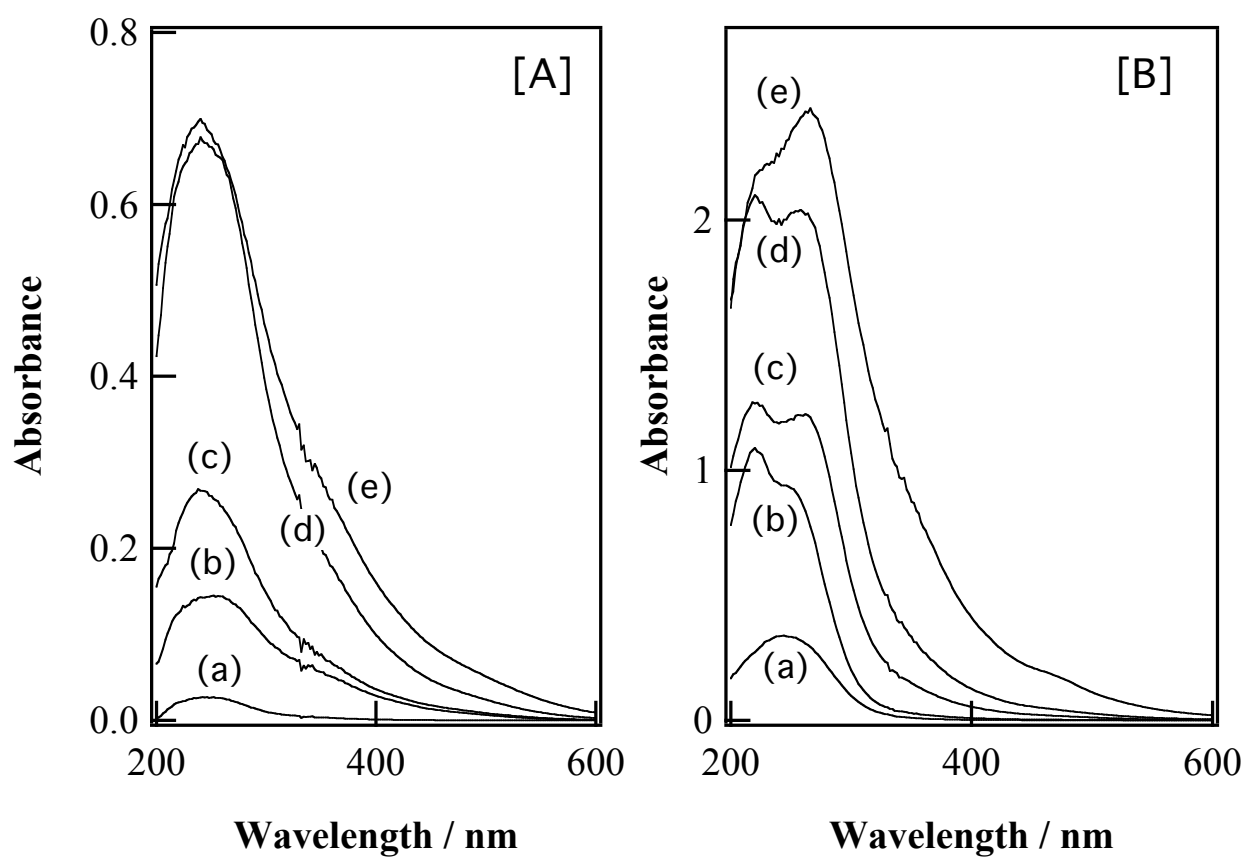

Figure 1

"Stabilization of flavylium dye..." by Yoshiumi Kohno 

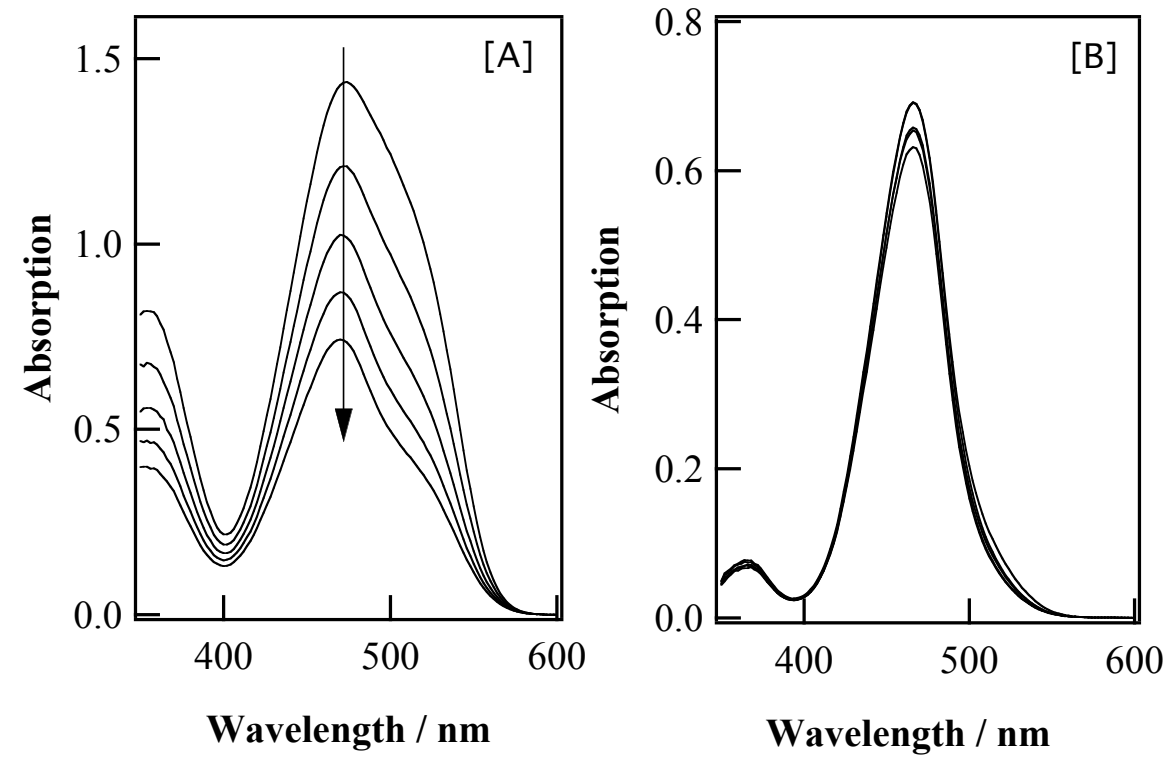

Figure 2

"Stabilization of flavylium dye..." by Yoshiumi Kohno 


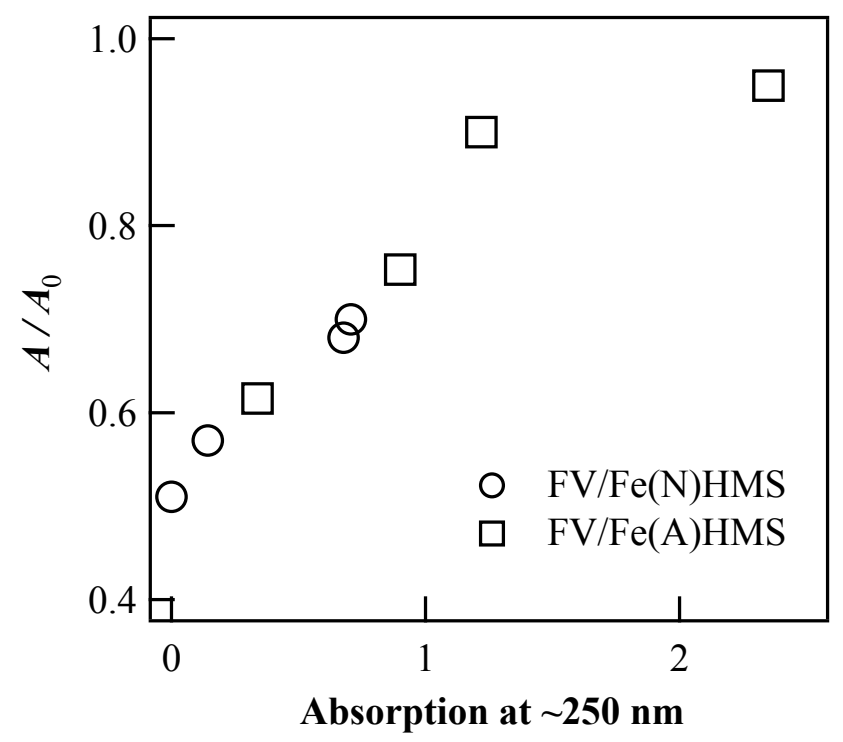

Figure 3

"Stabilization of flavylium dye..." by Yoshiumi Kohno 


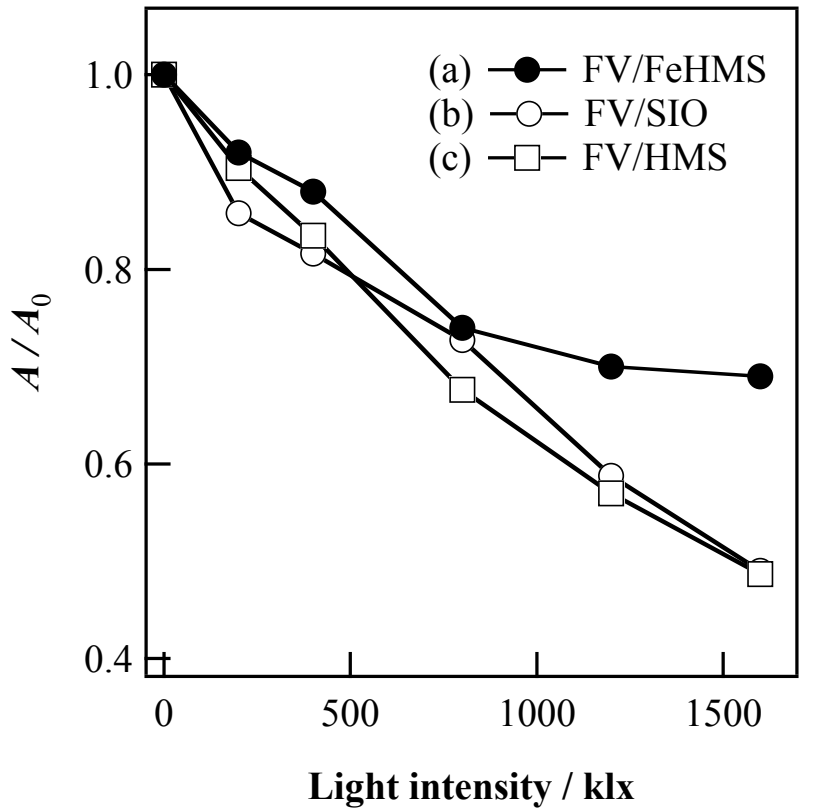

Figure 4

"Stabilization of flavylium dye..." by Yoshiumi Kohno 


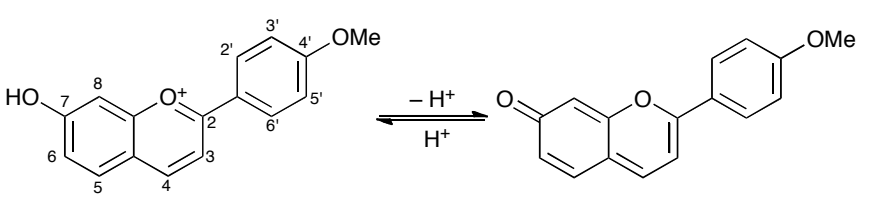

Scheme 1

"Stabilization of flavylium dye..." by Yoshiumi Kohno 